\title{
Mach cones in viscous heavy-ion collisions
}

\author{
I. Bouras, ${ }^{1}$ B. Betz, ${ }^{1}$ Z. Xu, ${ }^{2,3}$ and C. Greiner ${ }^{1}$ \\ ${ }^{1}$ Institut für Theoretische Physik, Johann Wolfgang Goethe-Universität, \\ Max-von-Laue-Str. 1, 60438 Frankfurt am Main, Germany \\ ${ }^{2}$ Department of Physics, Tsinghua University, Beijing 100084, China \\ ${ }^{3}$ Collaborative Innovation Center of Quantum Matter, Beijing 100871, China
}

(Dated: October 29, 2018)

\begin{abstract}
The formation of Mach cones is studied in a full $(3+1)$-dimensional setup of ultrarelativistic heavy-ion collisions, considering a transverse and longitudinal expanding medium at Relativistic Heavy-Ion Collider energies. For smooth initial conditions and central collisions the jet-medium interaction is investigated using high-energy jets and various values of the ratio of shear viscosity over entropy density, $\eta / s$. For small viscosities, the formation of Mach cones is proven, whereas for larger viscosities the characteristic structures smear out and vanish eventually. The formation of a double-peak structure both in a single- and in a multiple-jet event is discussed.
\end{abstract}

\section{INTRODUCTION}

Collisions of heavy nuclei both at the Relativistic Heavy-Ion Collider (RHIC) [14 and the Large Hadron Collider (LHC) [5] 8 indicate the formation of a new state of strongly interacting matter, the quark-gluon plasma (QGP). The large value of the measured elliptic flow coefficient, $v_{2}$, suggests that the QGP behaves like an almost perfect fluid as demonstrated by various calculations of viscous hydrodynamics 912 and microscopic transport models 13 15. The values determined for the ratio of shear viscosity over entropy density are approximately $\eta / s=0.1-0.2$ and thus close to the conjectured lower bound, $\eta / s=1 / 4 \pi$, derived via the correspondence between conformal field theory and string theory in an anti-de Sitter (AdS) space [16].

High-energy jets traversing the expanding medium deposit energy and momentum. Because of this interaction with the bulk medium, those jets are (strongly) suppressed, a phenomenon that is called jet quenching [1 4, 17 21]. The explicit mechanisms of the jetmedium interaction are still a matter of research. The two- and three-particle correlations extracted from experiment show a complete suppression of the away-side jets for $3<p_{T}<6 \mathrm{GeV}$ and due to energy and momentum conservation the signal appears again in a lower- $p_{T}$ range, showing a double-peak structure 22 27. The origin of the double-peak structure was discussed to be connected to Mach cones generated by fast partons traversing the strongly coupled medium [28 47]. However, recent studies on the triangular flow and hot spots 22, 48,54 issued a further, probably more satisfactory explanation, for the appearance of the double-peak structure.

Up until today, however, the contribution of jetinduced Mach cones to the double-peak structure remains unclear. To gain a better understanding of the contribution of Mach cones to the double-peak structure in twoparticle correlations, we investigate the evolution pattern of jet-induced Mach cones and their particle distribution in a relativistic, $(3+1)$-dimensional expanding system of a heavy-ion collision for various values of the ratio of shear viscosity over entropy density, $\eta / s$, using the microscopic transport model BAMPS (Boltzmann approach of multiparton scatterings) [55].

In an earlier publication [56, we already studied the formation of Mach cones using the above-mentioned kinetic transport model applying a static box scenario and thus neglecting all effects from expansion. We showed that, considering a source term depositing both energy and momentum, the double-peak structure is overshadowed by the strong contribution of the head shock and diffusion wake, while the double-peak structure appears for a source term (that lacks physical motivation from any theoretical model) which leads to only energy but no momentum deposition. These results are consistent with an investigation based on an AdS/Conformal Field Theory calculation [35] and a hydrodynamic model [57, 58. The comparison with the latter publications proves once more that BAMPS can model a hydrodynamic expansion [59, 60]. Using the same non-expanding scenario, we studied the effect of dissipation in a box scenario [56 and found that dissipation tends to destroy any Mach cone signal.

However, as discussed in Ref. 61, the flow-velocity profile created by jets in the transverse plane interacts with the radial flow of the background medium, changing, e.g., the effective angle of a Mach cone. This effect of transverse expansion on Mach cones has also been studied in Refs. [46]. It was found that a double-peak structure can be created by averaging over different jet paths. The main contribution to the double-peak structure originates from jets that are deflected by radial flow. In the rare case that a jet traverses into the opposite direction of the radial flow, the interplay between radial flow and jets reduces the strong contribution of the diffusion wake and head shock and results in a double-peak structure for this single event.

In the following, we investigate a similar setup as in Ref. [46, but including not only transverse but also longitudinal expansion, exploring in particular the influence of viscosity. In this work, the units are $\hbar=c=k=1$. 


\section{NUMERICAL SETUP}

We use the framework of BAMPS in a fully $(3+1)$ dimensional setup designed for ultrarelativistic heavy-ion collisions [55. Here, we treat a massless gluon gas as classical Boltzmann particles with a degeneracy factor of $g=16$. Particles only collide via binary collisions with an isotropic cross section, i.e., a cross section with an isotropic distribution of the collision angle. We perform the numerical calculations using a constant value of the ratio of shear viscosity over entropy density, $\eta / s$. For isotropic binary collisions, the shear viscosity is given by $\eta=0.4 e /(n \sigma)$ 62, where $e(n)$ is the local rest frame energy (particle) density.

As we intend to focus on the impact of transverse radial and longitudinal flow on jets, we neglect additional effects from density fluctuations. Likewise, we neglect any effects originating from elliptic flow and only consider central collisions. We apply smooth Glauber initial conditions in the transverse direction 63 and a Gaussian rapidity distribution in the longitudinal direction as it was found at RHIC 64 that the rapidity distribution of charged hadrons can be described by a Gaussian. In the beam $(z)$ direction, we determine the width of the nuclear overlap region based on the Lorentz-contracted nuclear thickness that is approximated by a Gaussian as well. (Please note that we initialize the system with a finite width. The system, however, will behave boostinvariantly near $z=0$ at times $<1 \mathrm{fm}$.) For the high$p_{T}$ region we apply a power law which approximately fits the $p+p$ data 65, 66. Thus, we apply the following parametrization for the initial non-thermal singleparticle distribution function

$$
\begin{aligned}
f(\vec{x}, \vec{p})= & K \frac{1}{E}\left(\frac{Q^{n}}{Q^{n}+p_{T}^{n}}\right)^{m} \exp \left(-\frac{y_{\text {rap }}^{2}}{\sigma_{y}^{2}}\right) \\
& \times \exp \left(-\frac{z^{2}}{\sigma_{z}^{2}}\right) T_{\mathrm{A}}\left(x+\frac{b}{2}, y\right) T_{\mathrm{B}}\left(x-\frac{b}{2}, y\right),
\end{aligned}
$$

where $p_{T}=\sqrt{p_{x}^{2}+p_{y}^{2}}$ denotes the transverse momentum, $y_{\text {rap }}$ the momentum rapidity, and $b$ the impact parameter. The nuclear thickness function [67]

$$
T_{\mathrm{A}}(x, y)=\int_{-\infty}^{+\infty} d z \rho_{\mathrm{A}}(x, y, z)
$$

is integrated in the $z$-direction. $\rho_{\mathrm{A}}(\vec{x})$ denotes the Woods-Saxon distribution for the nucleus $A$,

$$
\rho_{\mathrm{A}}(\vec{x})=\frac{\rho_{0}}{1+\exp \left(\frac{|\vec{x}|-R_{\mathrm{A}}}{D}\right)} .
$$

Here, $R_{\mathrm{A}}=1.12 A^{1 / 3}-0.86 A^{-1 / 3}$ and the mean density of the nucleus is $\rho_{0}=0.17 \mathrm{fm}^{-3}$. For the thickness parameter we use $D=0.54 \mathrm{fm}$. As we are studying $\mathrm{RHIC}$

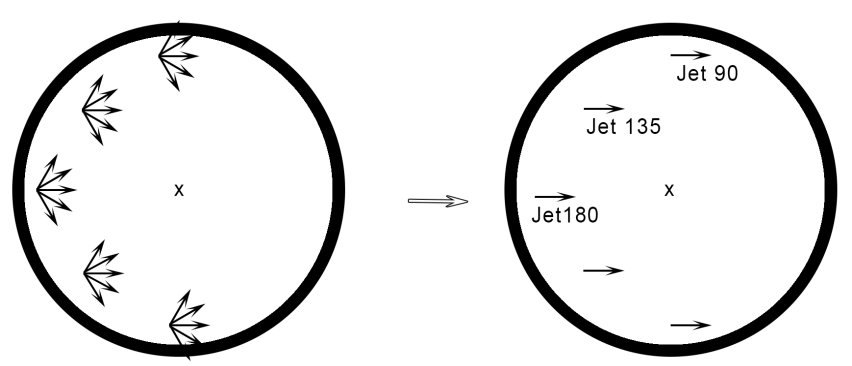

FIG. 1. (Color online) Schematic representation of different jet paths initialized on a semi-circle; see also Ref. [46. The left-hand panel shows all possible paths, and the right-hand panel shows the reduced number of paths due to reasons of symmetry.

energies, we exclusively use gold nuclei with a mass number of $A=B=197$ and choose $Q=1.3 \mathrm{GeV}, n=4$, $m=1.5, \sigma_{y}=1, \sigma_{z}=0.13 \mathrm{fm}$, and $K=0.0135$.

In the following we investigate scenarios where the jet is set on top of the bulk medium. Please note that the jet interacts with the same cross section as the medium particles.

Similar to the numerical setup discussed in Ref. 46] we initialize the starting points of the jet on a semi-circle displayed in Fig. 1 with a radius of $r=4 \mathrm{fm}$ at midrapidity,

$$
\vec{x}_{\mathrm{jet}}=r\left(\begin{array}{c}
\cos \phi_{\mathrm{jet}} \\
\sin \phi_{\mathrm{jet}} \\
0
\end{array}\right) \text {. }
$$

While in the experiment back-to-back correlated jets are created due to momentum conservation, we assume that the near-side jet escapes rapidly to the vacuum and thus, we neglect the near-side jet contribution. In contrast, the away-side jet traverses the hot and dense medium of the collision.

Because of symmetry, the possible jet paths that need to be studied in a central collision (see left-hand panel of Fig. 1) reduces drastically (as shown in the right-hand panel of Fig. 11. All jets studied here have an initial momentum only in the $x$-direction of $p_{x}=E_{\text {jet }}=20$ $\mathrm{GeV}$.

Although performing a full $(3+1)$-dimensional simulation, we restrict the following discussion on the midrapidity region. Thus, we extract all quantities and azimuthal particle distributions for a small space-time rapidity of $\left|\eta_{\text {rap }}\right|<0.1$. Since the parton cascade BAMPS has no effective hadronization process implemented yet, the final particle distribution is obtained by stopping the simulation at a certain time and extracting the macroscopic quantities as well as the azimuthal particle distribution from the final gluon-momentum distribution. 


\section{RESULTS}

In the following we study the evolution of jet-induced Mach cones in ultrarelativistic heavy-ion collisions for three different scenarios in order to gain a better understanding of the contribution of transverse and longitudinal expansion on the evolution of Mach cones induced by high-energy jets, considering various values of the ratio of shear viscosity over entropy density, $\eta / s$.

\section{A. Scenario I}

In a first scenario we consider a jet starting at a fixedangle position of $\phi_{\text {jet }}=180^{\circ}$ on a semi-circle. In this particular case the jet initially propagates in the opposite direction to the radial flow generated in a heavy-ion collision. We examine different values of the ratio of shear viscosity over entropy density, reflecting different possible interaction strengths with the medium. To illustrate the results we depict a time evolution of the local-rest frame energy-density profile around mid-rapidity, $\left|\eta_{\text {rap }}\right|<0.1$, in Fig. 2, overlaid with a velocity profile represented by scaled arrows.

At early times, $t \sim 1 \mathrm{fm} / c$, the energy density reaches very high values and a Mach cone has not yet built up. The energy behind the jet, however, is significantly lowered for $\eta / s=0.08$, indicating that matter is pushed in the forward direction. This effect is reduced with increasing values of $\eta / s$.

At later times, the energy density decreases drastically due to the longitudinal and the transverse expansion. A conical structure induced by the jet has developed at $t=5 \mathrm{fm} / c$ for $\eta / s=0.08$. In contrast, for larger values of $\eta / s$, such a structure has not built up (yet). The differences in the shape of the Mach cone for various values of $\eta / s$ becomes more evident at later times, $t=9$ and $t=12 \mathrm{fm} / c$. In the case of $\eta / s=0.08$, the energy density in the region of the developed shock front is much more increased than for larger values of $\eta / s$. Interestingly enough, the maximal energy density reached in the head-shock region for $\eta / s=0.2$ is smaller than for $\eta / s=0.5$. The reason is that the interaction of the jet with the medium is weakened for $\eta / s=0.5$; i.e., the jet is almost not quenched. In turn this means that the jet propagates faster for larger values of $\eta / s$. For the case with $\eta / s=0.08$ the initial jet has already lost almost all of its energy, i.e., has thermalized, within a very short time in the beginning of the simulation. This effect is demonstrated in Fig. 3, where the corresponding time evolution of the jet energy for different values of $\eta / s$ is shown. As mentioned above we do not distinguish between bulk medium and jet particles, which implies that for $\eta / s=0.08$ the mean free path of the jet is very small. On the other side, the larger the $\eta / s$, the smaller is the energy loss of the jet. It is clear that considering perturbative QCD based elastic and inelastic processes [68, 69 would change significantly the jet energy loss. However, such a treatment remains a future task.

The results in Fig. 2 indicate that a single jet propagating through the middle of the medium creates a Mach cone for moderate values of $\eta / s$ and sufficiently large times. The shock front, however, is strongly curved due to the interaction of the jet with the medium. The diffusion wake is not directly visible as it is superimposed by the radial flow of the background medium, indicating that the contribution of the diffusion wake and head shock is possibly reduced in the final particle distribution. After passing the center of the collision, however, the radial flow broadens the jet-induced shock front region. Please note that there are two competing effects here. As shown in Fig. 4 at $t=5 \mathrm{fm} / c$, the radial flow increases with larger $\eta / s$, while the interaction of the jet with the background medium decreases for larger $\eta / s$. Thus, the strength of the radial flow changes between the different $\eta / s$ scenarios studied here.

In Fig. 5, we show the extracted normalized azimuthal particle distribution $\mathrm{d} N /(N \mathrm{~d} \phi)$. The plot demonstrates that a double-peak structure can develop for a small value of the ratio of shear viscosity over entropy density, $\eta / s$, and a $p_{T}$-cut of $p_{T}>0.5 \mathrm{GeV}$. For $\eta / s=0.08$ and $0.5<p_{T}<1 \mathrm{GeV}$ a double-peak structure is observed at $\phi \approx \pm 70^{\circ}$, suggesting that the contribution of head shock and diffusion wake is indeed compensated by the radial flow. For larger transverse momenta, however [see, e.g., Fig. 5 (d)], the double-peak structure appears around $\phi \approx \pm 120^{\circ}$. Here, this conical structure is due to the fact that matter flows into a region of lower pressure and energy density behind the jet and is thus not directly a signal of the Mach cone. Finally, for $\eta / s=0.5$ the contribution from the head shock and diffusion wake is very strong, leading to a single peak in the forward direction.

The above discussion reveals that a Mach cone can only develop if the energy of the jet is neither too high nor too low. In the former case, the strong contribution of the head shock and diffusion wake leads to a single peak in the jet direction, whereas in the latter case the radial flow of the background medium distorts a Mach cone signal.

Please note that the local energy density in Fig. 2 at $t=12 \mathrm{fm} / c$ is much smaller than the typically expected $e_{\text {crit }} \approx 0.6 \mathrm{GeV} / \mathrm{fm}^{3}$ in the deconfined phase 14. We mentioned above that BAMPS has no effective hadronization procedure; thus, we extract all observables from the final gluon distribution. However, Fig. 6 proves that the extracted azimuthal two-particle correlations do not change significantly after $t=5-6 \mathrm{fm} / c$. While the cross section increases to unphysical values for energy densities smaller than the critical energy density $e_{\text {crit }} \approx 0.6 \mathrm{GeV} / \mathrm{fm}^{3}$, those energy densities are thus so small that the interaction rate of the system decreases rapidly. This implies that the further evolution of the deconfined phase with the same $\eta / s$ after $t=5-6 \mathrm{fm} / c$ is reasonable. 


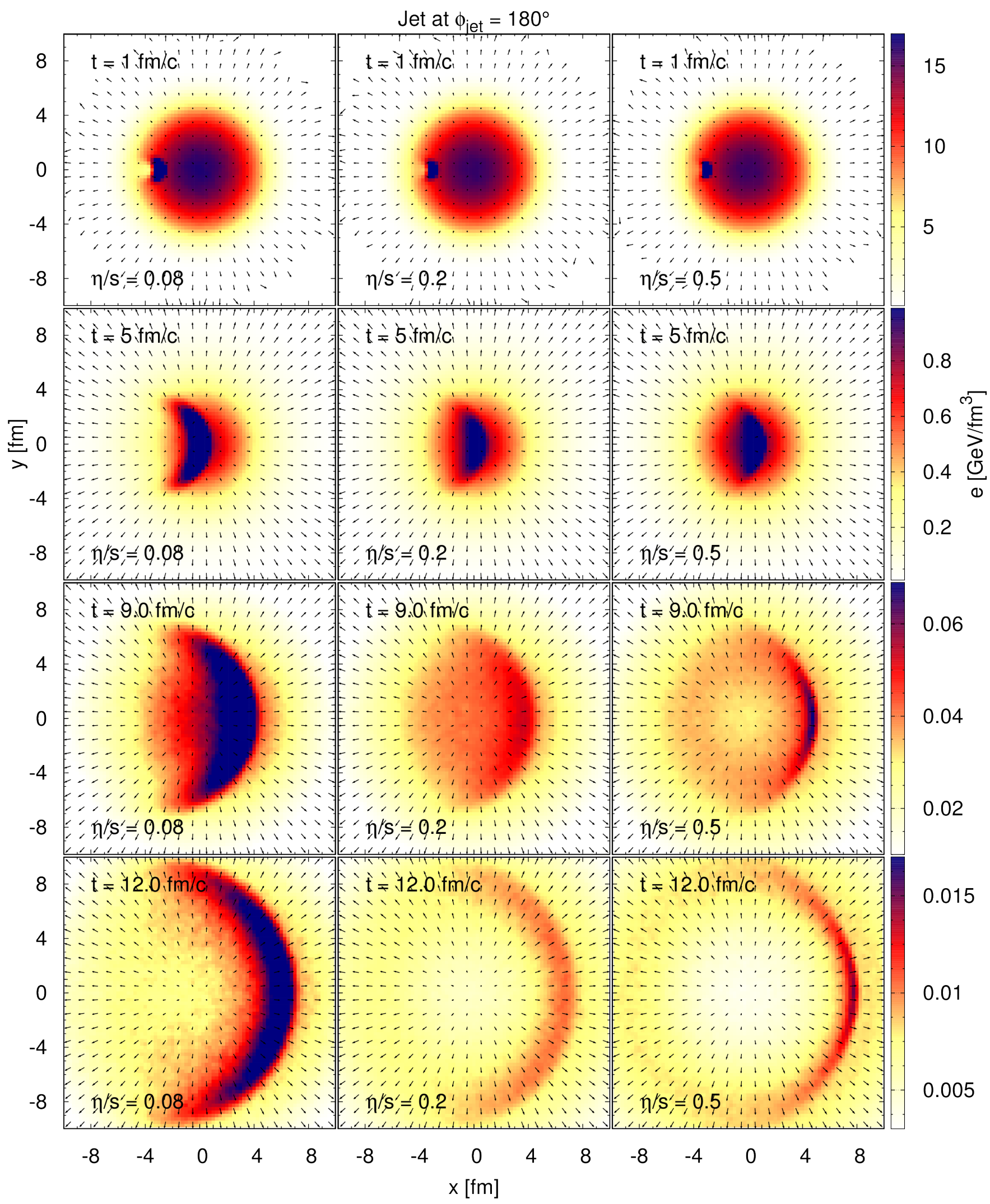

FIG. 2. (Color online) Time evolution of a Mach cone in a central heavy-ion collision. The local rest frame energy density around midrapidity $\left(\left|\eta_{\text {rap }}\right|<0.1\right)$ is shown, overlaid by the velocity profile, indicated by scaled arrows. The results are depicted at various time steps and different values of the ratio of shear viscosity over entropy density, $\eta / s$. The jet is initialized at a fixed-angle position of $\phi_{\text {jet }}=180^{\circ}$ on the semi-circle with an initial momentum of $p_{x}=E_{\text {jet }}=20 \mathrm{GeV}$. 


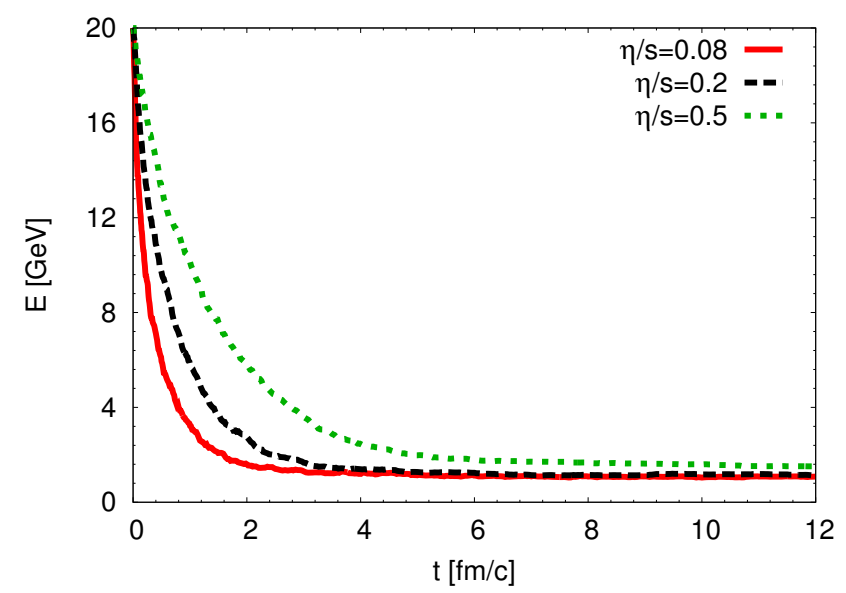

FIG. 3. (Color online) The time evolution of the jet energy extracted from BAMPS calculations shown in Fig. 2 for different values of the ratio of shear viscosity over entropy density, $\eta / s$.

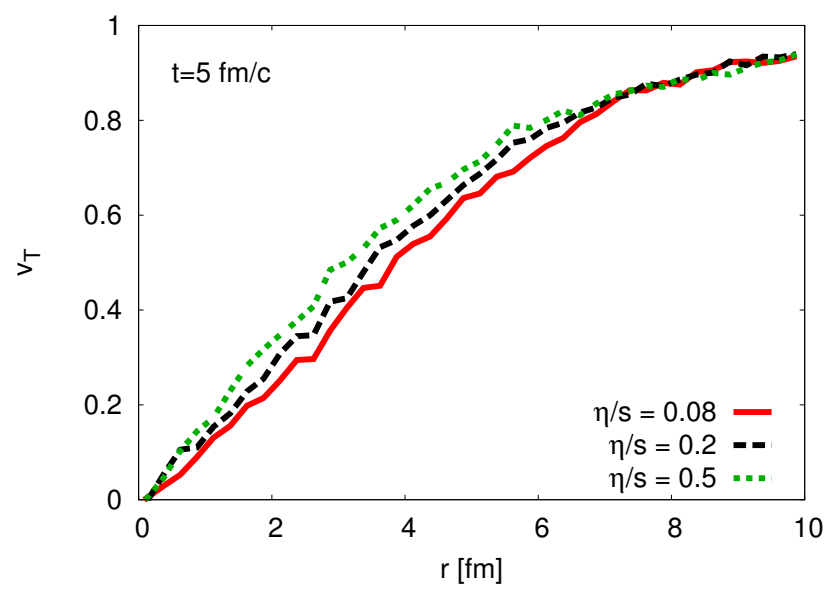

FIG. 4. (Color online) The extracted radial flow from BAMPS calculations at $t=5 \mathrm{fm} / c$ and central collisions for different values of the ratio of shear viscosity over entropy density, $\eta / s$.

\section{B. Scenario II}

In a second scenario, we consider a single jet with a fixed-angle position of $\phi_{\text {jet }}=135^{\circ}$ on the semi-circle to study the effects of deflection on the jet due to radial flow. The time evolution for different values of the ratio of shear viscosity over entropy density, $\eta / s$ is depicted in Fig. 7. Due to the relative position on the semi-circle and the strong radial flow, the single jet is deflected, especially for small $\eta / s$. This deflection, however, reduces for large $\eta / s$ as the jet-medium interaction weakens, keeping the jet on its initial propagation direction.

A direct comparison to scenario I indicates that the jet traverses a significantly lower energy-density region,
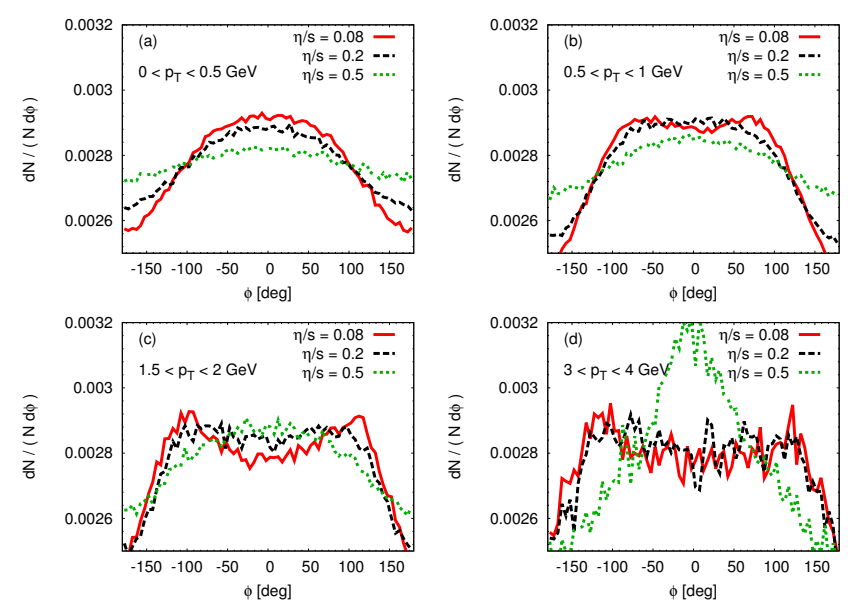

FIG. 5. (Color online) Two-particle correlations, $\mathrm{d} N /(N \mathrm{~d} \phi)$, extracted from BAMPS calculations for different values of $\eta / s$ and $p_{T}$-cuts. The jet is initialized at a fixed-angle position of $\phi_{\text {jet }}=180^{\circ}$ on the semi-circle (see Fig. 2). The results are shown for a fixed time $t=12 \mathrm{fm} / c$.
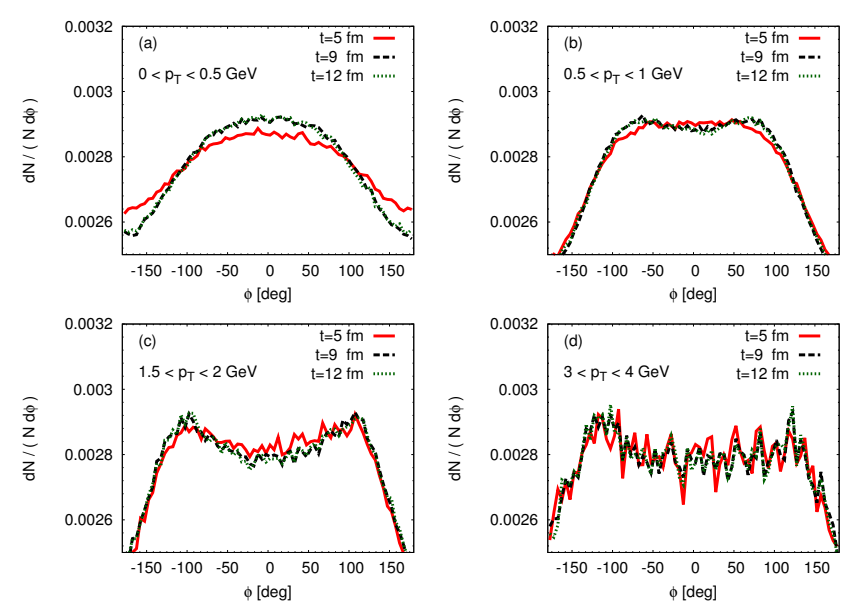

FIG. 6. (Color online) Two-particle correlations, $\mathrm{d} N /(N \mathrm{~d} \phi)$, extracted from BAMPS calculations for a fixed value of $\eta / s=$ 0.08 and various evolution times. The jet is initialized at a fixed-angle position of $\phi_{\text {jet }}=180^{\circ}$ on the semi-circle (see Fig. 2).

thus reaching only much lower values for the energy density in the shock-front region, especially for $\eta / s=0.08$. For lower energy-density regions, however, the mean free path of the jet is larger and results in a reduced jet quenching. However, a medium with $\eta / s=0.08$ is characterized by a strong collective behavior. The radial flow leads to a strongly distorted Mach cone pattern which is significantly reduced in a medium with $\eta / s=0.5$.

These results indicate that the generated diffusion wake and head shock are less compensated by the radial flow in scenario II due to the fact that the jet does not propagate in the direction opposite to the radial flow. 


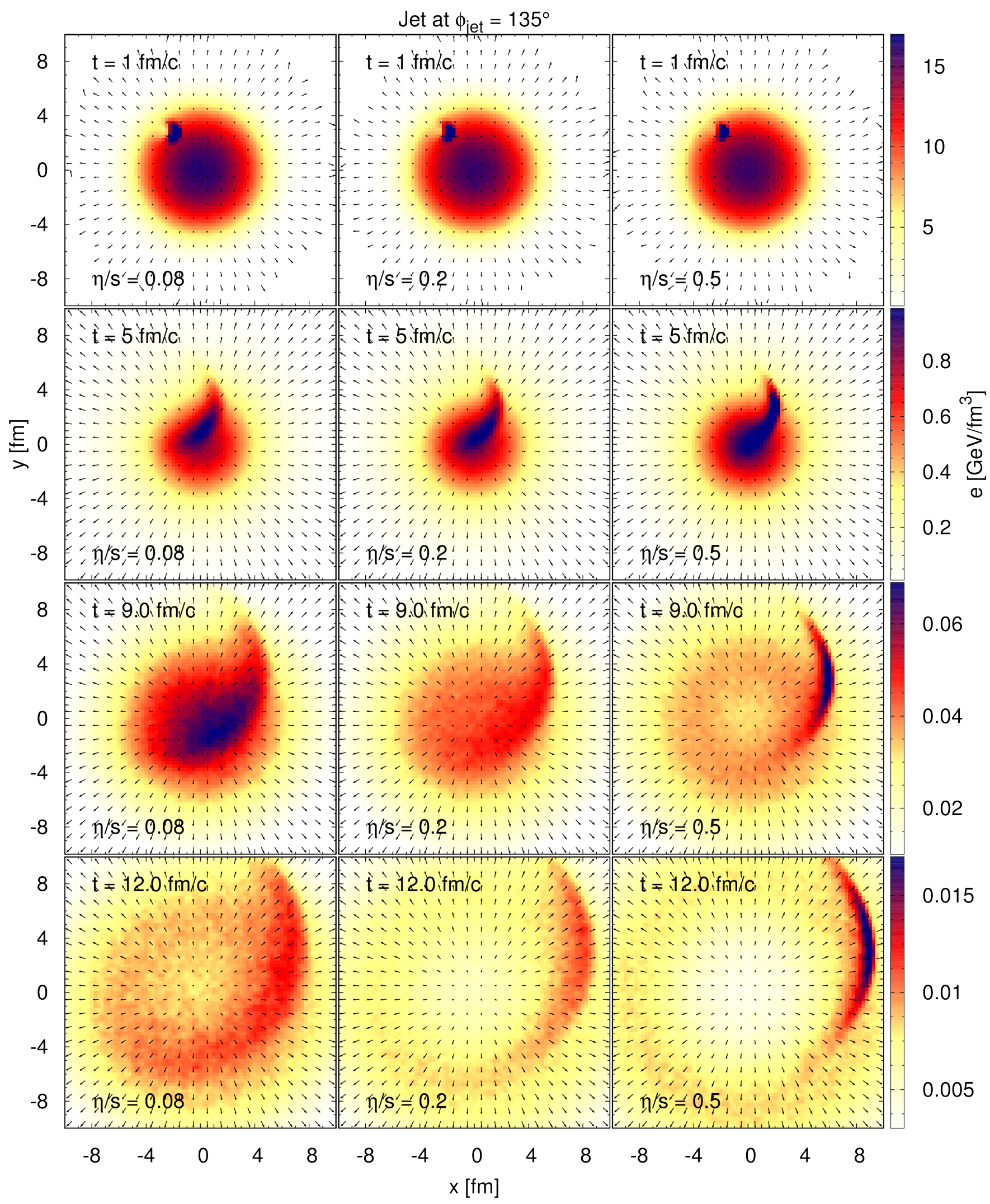

FIG. 7. (Color online) Time evolution of a Mach cone in a central heavy-ion collision. We show the local-rest frame energy density within a mid-rapidity range of $\left|\eta_{\text {rap }}\right|<0.1$, overlaid by the velocity profile indicated by scaled arrows. The results are depicted at various time steps and for different values of the ratio of shear viscosity over entropy density, $\eta / s$. The jet is initialized at a fixed-angle position of $\phi_{\text {jet }}=135^{\circ}$ on the semi-circle with an initial momentum of $p_{x}=E_{\text {jet }}=20 \mathrm{GeV}$. 

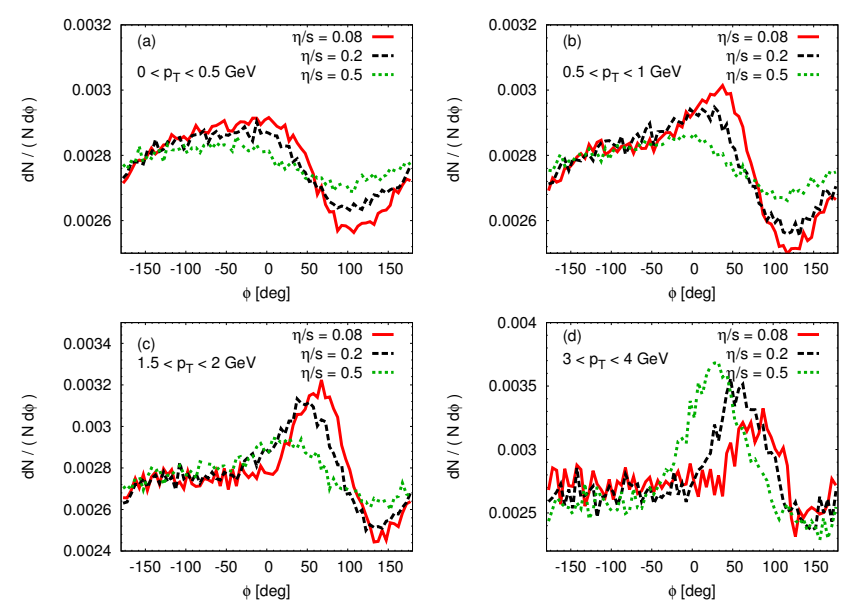

FIG. 8. (Color online) Two-particle correlations, $\mathrm{d} N /(N \mathrm{~d} \phi)$, extracted from BAMPS calculations for different values of $\eta / s$ and $p_{T}$-cuts. The jet is initialized at a fixed-angle position of $\phi_{\text {jet }}=135^{\circ}$ on the semi-circle (see Fig. 7). The results are shown for a fixed time $t=12 \mathrm{fm} / c$.

The deflection of the jet for $\eta / s=0.08$ leads to only one peak in the two-particle correlations as displayed in Fig. 8. This peak originates from the head shock and diffusion wake of the distorted Mach cone and becomes sharper and more pronounced for larger $p_{T}$. We also see that the position of the induced peaks in Fig. 8 changes with viscosity. The larger the value of $\eta / s$, the smaller the peak angle, reflecting the fact that the jet is less deflected for larger values of $\eta / s$.

Although the two-particle correlations determined for this specific single-jet scenario only exhibit one single peak, a double-peak structure is possible when considering a second jet event in the lower semi-circle [46, 7072. In this case, however, the double peak is mainly generated due to the superposition of several deflected and distorted jet-induced Mach cones; i.e., the origin for the double-peak structure is not the Mach cone itself but the deflected head shocks and diffusion wakes of the distorted Mach cones as demonstrated below in scenario III.

\section{Scenario III}

Averaging over all possible jet trajectories with randomly chosen starting positions on the semi-circle, i.e., $\phi_{\text {jet }}=90-270^{\circ}$, scenario III gets closest to the experimental situation. Here, in contrast to scenarios I and II, we consider many different jet events. Figure 9 displays the normalized azimuthal particle distribution determined for this multiple-jet scenario. A double-peak structure appears for sufficiently high $p_{T}$ and low $\eta / s$ with peaks around $\phi \approx 50^{\circ}$. This result is in line with Ref. [46] applying a hydrodynamic evolution.
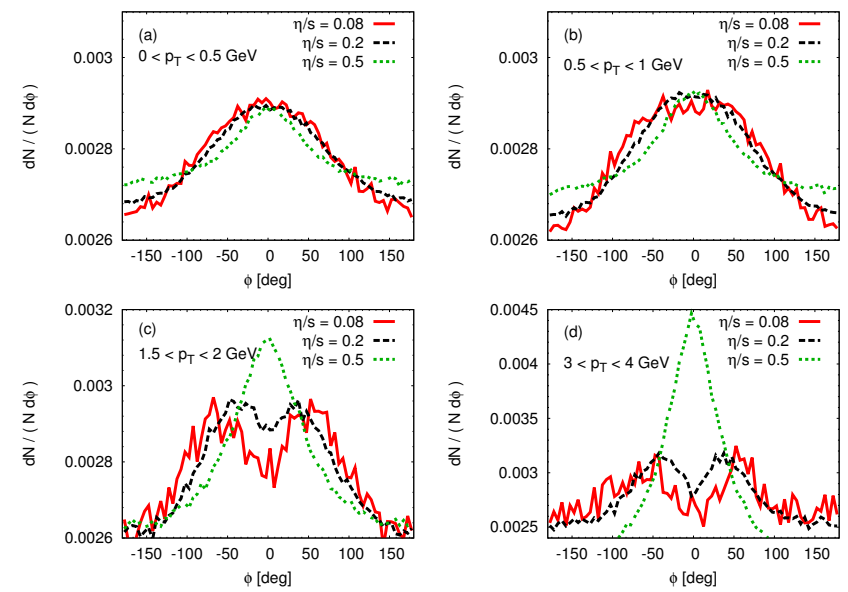

FIG. 9. (Color online) Two-particle correlations, $\mathrm{d} N /(N \mathrm{~d} \phi)$, extracted from BAMPS calculations for different values of $\eta / s$ and $p_{T}$-cuts. Here, we consider various starting positions of the jet on the semi-circle. The results are shown at a fixed time of $t=12 \mathrm{fm} / c$.

\section{DISCUSSION}

The above results provide evidence that Mach cones can form in a heavy-ion collision if the medium created has a small viscosity. However, as compared to an ideal, zero-viscosity medium, the pattern of the Mach cone is more curved. If a jet gets deflected by radial flow, the shape of the induced Mach cone is distorted. We demonstrate that the radial flow in a heavy-ion collision affects both, the final pattern of the Mach cone 61 and the observed particle distribution.

Considering a single jet event for a sufficiently small value of $\eta / s=0.08$, the interplay of jet-induced Mach cones and radial flow may lead to a double-peak structure, as shown in scenario I. In this scenario the doublepeak structure originates from the Mach cone since the contributions of head shock and diffusion wake are canceled by the radial flow.

For a single jet not traversing the medium in direction opposite to the radial flow, however, the doublepeak structure does not appear; cf. scenario II. Here, the radial flow leads to a deflection of the jet, resulting in a single peak arising in the two-particle correlations. Nonetheless, considering a multiple-jet scenario as in scenario III, a double-peak structure occurs as well that is mainly generated by the superposition of the deflected and distorted head shocks and diffusion wakes of the jetinduced Mach cones [46, 70].

We demonstrate above that a sufficiently large value of $\eta / s$ tends to destroy the characteristic structure of a Mach cone in a three-dimensional expanding heavyion collision and results in a single peak in the twoparticle correlation. Here, the contributions from both head shock and diffusion wake are less canceled by the 
radial flow than in the case of a smaller value of $\eta / s$. Moreover, larger dissipation reduces the deflection of jets. However, not only viscosity tends to destroy the doublepeak structure, but also the strength of the jet. A jet with a large initial energy punches through the medium and the effect of the radial flow weakens. For such a highenergy jet, the diffusion wake and head-shock contribution in scenario I would overshadow the double-peak structure, while in scenario III most of the jets would get less deflected. However, a jet with a very small initial energy density would not lead to a double-peak structure either, as in this case the strong radial flow observed in a heavy-ion collision does not allow a Mach cone to be developed.

Our results imply that the influence of the radial flow is most important for the observation of a double-peak structure. A large dissipation and/or a large initial jet energy leads to a smaller influence of the radial flow. The effect of longitudinal flow is studied here in a qualitative way and was not considered in previous studies [46. We conclude that the longitudinal flow does not affect the results significantly.

The Mach-cone angle extracted from two-particle correlations is certainly not connected to the equation-of state [61. Scenario III provides evidence that the contribution of this double-peak structure mainly originates from the head shocks and diffusion wakes of the jetinduced Mach cones. To extract the Mach angle and thus determine the speed of sound of the medium would require the measurement of a single jet event as shown in scenario I which remains an extremely tough experimental challenge.

\section{CONCLUSION}

In conclusion, we have shown that Mach cones can form in a longitudinal and transverse expanding heavy- ion collision. Considering multiple-jet events, a doublepeak structure evolves if the ratio of shear viscosity over entropy density is $\eta / s<0.5$. Please note that the peak position does not change significantly with $\eta / s$. However, this double-peak structure does not originate from Mach cones but arises due to the contribution of the head shock and diffusion wake originating from the deflected jets 46 , 61.

Although initial-state fluctuations certainly provide a large contribution to the experimentally observed doublepeak structure [48 51, 73, 76, it will be important to quantify the contribution from jets. The double-peak signal might not be an appropriate observable to disentangle both contributions [56] as it is mainly medium driven. However, allocating a more appropriate signature remains an open challenge.

\section{ACKNOWLEDGEMENTS}

The authors are grateful to H. Niemi, E. Molnar, D. H. Rischke, J. Noronha, G. Torrieri, P. Huovinen, J. Ulery, and H. Stöcker for discussions and to the Center for Scientific Computing (CSC) at Frankfurt University for providing the necessary computing resources. I.B. acknowledges support from HGS-Hire. Z.X. work is supported partially by the Major State Basic Research Development Program in China (Grant No. 2014CB845400) and by the National Natural Science Foundation of China projects (Grants No. 11275103, 11335005). This work was supported by BMBF and the Helmholtz International Center for FAIR within the framework of the LOEWE program launched by the State of Hesse.
[1] J. Adams et al. (STAR), Nucl.Phys. A757, 102 (2005), arXiv:nucl-ex/0501009 [nucl-ex].

[2] I. Arsene et al. (BRAHMS), Nucl.Phys. A757, 1 (2005) arXiv:nucl-ex/0410020 [nucl-ex]

[3] K. Adcox et al. (PHENIX), Nucl.Phys. A757, 184 (2005), arXiv:nucl-ex/0410003 [nucl-ex].

[4] B. Back, M. Baker, M. Ballintijn, D. Barton, B. Becker, et al. (PHOBOS), Nucl.Phys. A757, 28 (2005), arXiv:nucl-ex/0410022 [nucl-ex].

[5] K. Aamodt et al. (ALICE), Phys. Rev. Lett. 105, 252301 (2010)

[6] K. Aamodt et al. (ALICE), Phys. Rev. Lett. 105, 252302 (2010)

[7] K. Aamodt et al. (ALICE), Phys. Rev. Lett. 106, 032301 (2011)

[8] K. Aamodt et al. (ALICE), Phys. Rev. Lett. 107, 032301 (2011)
[9] M. Luzum and P. Romatschke, Phys.Rev. C78, 034915 (2008), arXiv:0804.4015 [nucl-th].

[10] H. Song and U. W. Heinz, J.Phys. G36, 064033 (2009) arXiv:0812.4274 [nucl-th].

[11] H. Niemi, G. S. Denicol, P. Huovinen, E. Molnar, and D. H. Rischke, Phys.Rev.Lett. 106, 212302 (2011), arXiv:1101.2442 [nucl-th]

[12] B. Schenke, S. Jeon, and C. Gale, Phys.Lett. B702, 59 (2011), arXiv:1102.0575 [hep-ph].

[13] Z. Xu, C. Greiner, and H. Stoecker, Phys.Rev.Lett. 101, 082302 (2008), arXiv:0711.0961 [nucl-th].

[14] Z. Xu and C. Greiner, Phys.Rev. C79, 014904 (2009), arXiv:0811.2940 [hep-ph].

[15] J. Xu and C. M. Ko, Phys.Rev. C83, 034904 (2011) arXiv:1101.2231 [nucl-th]

[16] P. Kovtun, D. Son, and A. Starinets, Phys.Rev.Lett. 94, 111601 (2005), arXiv:hep-th/0405231 [hep-th] 
[17] J. Adams et al. (STAR), Phys.Rev.Lett. 91, 172302 (2003), arXiv:nucl-ex/0305015 [nucl-ex].

[18] J. D. Bjorken, Phys. Rev. D 27, 140 (1983).

[19] M. Gyulassy and M. Plumer, Nucl.Phys. B346, 1 (1990).

[20] X.-N. Wang and M. Gyulassy, Phys.Rev.Lett. 68, 1480 (1992)

[21] X.-N. Wang and M. Gyulassy, Nucl.Phys. A544, 559 (1992)

[22] F. Wang, Prog. Part. Nucl. Phys. 74, 35 (2014), arXiv:1311.4444 [nucl-ex]

[23] F. Wang (STAR), J.Phys. G30, S1299 (2004) arXiv:nucl-ex/0404010 [nucl-ex]

[24] J. Adams et al. (STAR), Phys.Rev.Lett. 95, 152301 (2005), arXiv:nucl-ex/0501016 [nucl-ex].

[25] S. Adler et al. (PHENIX), Phys.Rev.Lett. 97, 052301 (2006), arXiv:nucl-ex/0507004 [nucl-ex]

[26] J. G. Ulery (STAR), Nucl.Phys. A774, 581 (2006), arXiv:nucl-ex/0510055 [nucl-ex].

[27] N. Ajitanand (PHENIX), Nucl.Phys. A783, 519 (2007) arXiv:nucl-ex/0609038 [nucl-ex].

[28] H. Stoecker, Nucl.Phys. A750, 121 (2005), arXiv:nuclth/0406018 [nucl-th]

[29] W. Scheid, H. Mueller, and W. Greiner, Phys.Rev.Lett. 32, 741 (1974)

[30] J. Hofmann, H. Stoecker, U. W. Heinz, W. Scheid, and W. Greiner, Phys.Rev.Lett. 36, 88 (1976)

[31] H. Baumgardt, J. Schott, Y. Sakamoto, E. Schopper, H. Stoecker, et al., Z.Phys. A273, 359 (1975)

[32] H. Gutbrod, K. Kampert, B. Kolb, A. M. Poskanzer, H. Ritter, et al., Phys.Rev. C42, 640 (1990).

[33] R. Neufeld and I. Vitev, Phys.Rev. C86, 024905 (2012) arXiv:1105.2067 [hep-ph]

[34] R. Neufeld and T. Renk, Phys.Rev. C82, 044903 (2010), arXiv:1001.5068 [nucl-th]

[35] J. Noronha, M. Gyulassy, and G. Torrieri, Phys.Rev.Lett. 102, 102301 (2009), arXiv:0807.1038 [hep-ph]

[36] G. Ma, S. Zhang, Y. Ma, H. Huang, X. Cai, et al., Phys.Lett. B641, 362 (2006), arXiv:nucl-th/0601012 [nucl-th]

[37] I. Bouras, E. Molnar, H. Niemi, Z. Xu, A. El, et al., J.Phys.Conf.Ser. 230, 012045 (2010), arXiv:1004.4615 [hep-ph]

[38] J. Ruppert and B. Mueller, Phys.Lett. B618, 123 (2005) arXiv:hep-ph/0503158 [hep-ph]

[39] V. Koch, A. Majumder, and X.-N. Wang, Phys.Rev.Lett. 96, 172302 (2006), arXiv:nucl-th/0507063 [nucl-th].

[40] T. Renk and J. Ruppert, Phys.Rev. C73, 011901 (2006), arXiv:hep-ph/0509036 [hep-ph]

[41] S. S. Gubser, S. S. Pufu, and A. Yarom, Phys.Rev.Lett. 100, 012301 (2008), arXiv:0706.4307 [hep-th]

[42] J. Casalderrey-Solana, E. Shuryak, and D. Teaney, J.Phys.Conf.Ser. 27, 22 (2005), arXiv:hep-ph/0411315 [hep-ph].

[43] S. Zhang, G. Ma, Y. Ma, X. Cai, J. Chen, et al., Phys.Rev. C76, 014904 (2007), arXiv:0706.3820 [nuclth],

[44] W. Li, S. Zhang, Y. Ma, X. Cai, J. Chen, et al., Phys.Rev. C80, 064913 (2009)

[45] P. Rau, J. Steinheimer, B. Betz, H. Petersen, M. Bleicher, et al., (2010), arXiv:1003.1232 [nucl-th].

[46] B. Betz, J. Noronha, G. Torrieri, M. Gyulassy, and D. H. Rischke, Phys.Rev.Lett. 105, 222301 (2010), arXiv:1005.5461 [nucl-th].
[47] J. Casalderrey-Solana, J.Phys. G34, S345 (2007) arXiv:hep-ph/0701257 [HEP-PH]

[48] G.-L. Ma and X.-N. Wang, Phys.Rev.Lett. 106, 162301 (2011), arXiv:1011.5249 [nucl-th].

[49] B. Schenke, S. Jeon, and C. Gale, Phys.Rev.Lett. 106, 042301 (2011), arXiv:1009.3244 [hep-ph].

[50] R. S. Bhalerao, M. Luzum, and J.-Y. Ollitrault, Phys.Rev. C84, 054901 (2011), arXiv:1107.5485 [nuclth]

[51] J. Takahashi, B. Tavares, W. Qian, R. Andrade, F. Grassi, et al., Phys.Rev.Lett. 103, 242301 (2009), arXiv:0902.4870 [nucl-th]

[52] R. Andrade, F. Grassi, Y. Hama, and W.-L. Qian, J.Phys. G37, 094043 (2010), arXiv:0912.0703 [nucl-th],

[53] B. Alver and G. Roland, Phys.Rev. C81, 054905 (2010), arXiv:1003.0194 [nucl-th]

[54] M. Gyulassy, D. H. Rischke, and B. Zhang, Nucl.Phys. A613, 397 (1997), arXiv:nucl-th/9609030 [nucl-th].

[55] Z. Xu and C. Greiner, Phys.Rev. C71, 064901 (2005) arXiv:hep-ph/0406278 [hep-ph].

[56] I. Bouras, A. El, O. Fochler, H. Niemi, Z. Xu, et al., Phys.Lett. B710, 641 (2012), arXiv:1201.5005 [nucl-th]

[57] B. Betz, M. Gyulassy, J. Noronha, and G. Torrieri, Phys.Lett. B675, 340 (2009), arXiv:0807.4526 [hep-ph]

[58] B. Betz, J. Noronha, G. Torrieri, M. Gyulassy, I. Mishustin, et al., Phys.Rev. C79, 034902 (2009), arXiv:0812.4401 [nucl-th]

[59] I. Bouras, E. Molnar, H. Niemi, Z. Xu, A. El, et al., Phys.Rev. C82, 024910 (2010), arXiv:1006.0387 [hep$\mathrm{ph}]$,

[60] I. Bouras, E. Molnar, H. Niemi, Z. Xu, A. El, et al., Phys.Rev.Lett. 103, 032301 (2009), arXiv:0902.1927 [hep-ph]

[61] L. Satarov, H. Stoecker, and I. Mishustin, Phys.Lett. B627, 64 (2005), arXiv:hep-ph/0505245 [hep-ph].

[62] S. Groot, W. Leeuwen, and C. van Weert, Relativistic kinetic theory: principles and applications (North-Holland Pub. Co., 1980).

[63] M. L. Miller, K. Reygers, S. J. Sanders, and P. Steinberg, Ann.Rev.Nucl.Part.Sci. 57, 205 (2007), arXiv:nuclex/0701025 [nucl-ex]

[64] I. Bearden et al. (BRAHMS Collaboration), Phys.Rev.Lett. 94, 162301 (2005) arXiv:nuclex/0403050 [nucl-ex]

[65] S. Adler et al. (PHENIX), Phys.Rev.Lett. 91, 241803 (2003), arXiv:hep-ex/0304038 [hep-ex]

[66] J. Adams et al. (STAR), Phys.Rev. D74, 032006 (2006), arXiv:nucl-ex/0606028 [nucl-ex].

[67] P. Kolb, U. W. Heinz, P. Huovinen, K. Eskola, and K. Tuominen, Nucl.Phys. A696, 197 (2001), arXiv:hep$\mathrm{ph} / 0103234$ [hep-ph]

[68] O. Fochler, J. Uphoff, Z. Xu, and C. Greiner, (2013), arXiv:1302.5250 [hep-ph].

[69] O. Fochler, Z. Xu, and C. Greiner, Phys.Rev. C82, 024907 (2010), arXiv:1003.4380 [hep-ph].

[70] T. Renk and J. Ruppert, Phys.Lett. B646, 19 (2007), arXiv:hep-ph/0605330 [hep-ph].

[71] A. Chaudhuri, Phys.Rev. C75, 057902 (2007), arXiv:nucl-th/0610121 [nucl-th].

[72] A. Chaudhuri, Phys.Rev. C77, 027901 (2008), arXiv:0706.3958 [nucl-th]

[73] A. Ayala, I. Dominguez, and M. E. Tejeda-Yeomans, (2012), arXiv:1212.1127 [hep-ph]. 
[74] K. Aamodt et al. (ALICE Collaboration), Phys.Lett. B708, 249 (2012), arXiv:1109.2501 [nucl-ex]

[75] G. Aad et al. (ATLAS Collaboration), Phys.Rev. C86, 014907 (2012), arXiv:1203.3087 [hep-ex].
[76] S. Chatrchyan et al. (CMS Collaboration), Eur.Phys.J. C72, 2012 (2012), arXiv:1201.3158 [nucl-ex] 